\title{
Dynamic stabilization of a microwave-driven Josephson tunnel junction against a period-doubling bifurcation
}

Hansen, Jørn Bindslev; Eriksen, G. Friis; Samuelsen, Mogens Rugholm; Svensmark, H.

Published in:

I E E E Transactions on Magnetics

Link to article, DOI:

$10.1109 / 20.92559$

Publication date:

1989

Document Version

Publisher's PDF, also known as Version of record

Link back to DTU Orbit

Citation (APA):

Hansen, J. B., Eriksen, G. F., Samuelsen, M. R., \& Svensmark, H. (1989). Dynamic stabilization of a microwavedriven Josephson tunnel junction against a period-doubling bifurcation. I E E E Transactions on Magnetics, 25(2), 1408-1411. https://doi.org/10.1109/20.92559

\section{General rights}

Copyright and moral rights for the publications made accessible in the public portal are retained by the authors and/or other copyright owners and it is a condition of accessing publications that users recognise and abide by the legal requirements associated with these rights.

- Users may download and print one copy of any publication from the public portal for the purpose of private study or research.

- You may not further distribute the material or use it for any profit-making activity or commercial gain

- You may freely distribute the URL identifying the publication in the public portal 


\section{DYNAMIC STABILIZATION OF A MICROWAVE-DRIVEN JOSEPHSON \\ TUNNEL JUNCTION AGAINST A PERIOD-DOUBLING BIFURCATION}

J. Bindslev Hansen, G. Friis Eriksen, and M.R. Samuelsen.

Physics Laboratory I/MIDIT, The Technical University of Denmark.

DK-2800 Lyngby, Denmark.

and

H. Svensmark

Department of Physics,

University of California, Berkeley, California,

94720 USA.

Abstract

We present theoretical and experimental results on the effect of periodic perturbations on microwave-driven Josephson tunnel junctions close to a period-doubling bifurcation. The theory, which is quite general, describes how a periodic near-resonant perturbation changes the stability of the system. The phenomenon was investigated experimentally in small $\mathrm{Nb}-\mathrm{NbOx}-\mathrm{Pb}$ tunnel junctions and found to be in agreement with the theory. The effect has importance for the performance of parametric amplifiers based on Josephson tunnel junctions.

\section{Introduction}

The period-doubling bifurcation is a type of bifurcation commonly found in many biological, chemical, electrical, hydrodynamical, and optical systems. We here address the important question of the stability of a period-doubling bifurcation in the presence of perturbations with application to the Josephson tunnel junction ${ }^{1-5}$. We regard this analysis of the non-linear dynamics as a new way of describing the action of the Josephson parametric amplifier, a microwave device which has attracted renewed interest after recent reports on substantial improvements in performance ${ }^{6-7}$.

\section{Theoretical model}

This section summarizes the theory on the stability of the asymptotic behavior of a dynamical system which is close to a period-doubling bifurcation. A more thorough treatment is found in Refs. (9) and (10).

A general $\mathrm{n}$-dimensional dynamical system is assumed to be modelled by the following set of differential equations:

$$
\begin{aligned}
& \dot{x}=f_{\mu}(x, t)+s(t)+n(t), \\
&(x, t) \in \dot{R}^{n} \times \dot{R}, \quad \mu \in I
\end{aligned}
$$

where $f_{\mu}(\cdot, t)=f_{\mu}\left(\cdot, t+T_{D}\right)$ is periodic with the period $T_{D} . \mu$ is a control parameter which is defined in some interval I. $s(t)$ and $n(t)$ are a small signal (periodic perturbation) and a small noise term (stochastic perturbation), respectively. The period of the signal is denoted $T_{S}$ and the corresponding frequency $\omega_{S}=2 \pi / T_{S}$. The noise term $n(t)$ is assumed to be "white" defined by $\langle n(t) n(t+\tau)\rangle=\sigma^{2} \delta(\tau)$ and $\langle n(t)\rangle=0$. $|s(t)|$ and $|n(t)|$ are small compared to $\left|f_{\mu}(x, t)\right|$.

The unperturbed system is assumed to be close to a period-doubling bifurcation. By increasing the control parameter, the response will change from a periodic solution with period $T_{D}$ to a periodic solution of period $2 T_{D}$. The value of the control parameter for which this happens is called the bifurcation point.

The essential properties of a period-doubling bifurcation are: 1) at the bifurcation point the dynamics is confined to a surface in phase space called the center manifold; and 2) for a period-doubling bifurcation the center manifold is a two dimensional surface topologically equivalent to a Möbius band.

Following Ref.(8) we assume that close to the bifurcation point the dynamics in phase space are also confined to the center manifold. This means that the dynamics can be described by the following Poincaré map, regardless of the

Manuscript received August 22, 1988. original dimension of the dynamical system:

$$
x_{n+1}=P_{\mu}\left(x_{n}\right), x_{n} \in \grave{R}, \mu \in I .
$$

If $\mu$ is below the bifurcation point, the dynamical system given by Eq.(1) has a periodic solution of period $T_{D}$, which in the Poincare map corresponds to a fix point $x^{j i x}$. It is clear that the stability of the fix point reflects the stability of the periodic solution. The stability of the unperturbed dynamical system is therefore analyzed by linearizing the Poincare map around the fix point.

If we now allow a small periodic perturbation with the frequency $\omega_{S}$ (the term $s(t)$ in Eq.(1)) the function $P_{\mu}\left(x_{n}\right)$ in the Poincaré map (Eq.(2)) will change, since the terms on the right hand side of Eq.(1) no longer have the period $T_{D}$. So the Poincaré map becomes

$$
x_{n+1}=P_{n, \mu}\left(x_{n}\right) .
$$

Since the perturbation is small the change in the Poincaré map may be written as

$$
x_{n+1}=P^{\mu}\left(x_{n}\right)+\Delta P_{n}\left(x_{n}-x^{f i x}\right),
$$

where the first term, $P^{\mu}$, is the unperturbed Poincare map and the second term, $\Delta P_{n}$, is a small correction due to the perturbation. This last term changes for each iteration but has to be a periodic function which changes for integer intervals of $T_{S}$ according to the right hand side of Eq.(1).

At a given instant of time, the phase angles for the two first terms on the right hand side of Eq.(1) can be viewed as coordinates on a torus

$\left(\phi_{D}, \phi_{S}\right)=\left(\omega_{D} t \bmod 2 \pi, \omega_{S} t \bmod 2 \pi\right)$, as seen in Fig. 1 , which illustrates the important case where the ratio $\omega_{D} / \omega_{S}$ is close to $1 / 2$. After each iteration the phase angle will progress by $\pi \delta$. This example is easily generalized to any ratio of $\omega_{D} / \omega_{S}$ with $\delta$ given by

$$
\delta=2\left(\omega_{S} / \omega_{D}\right)-k,
$$

where $k$ is an integer chosen such that $-1 / 2<\delta<1 / 2$, and $\delta$ is

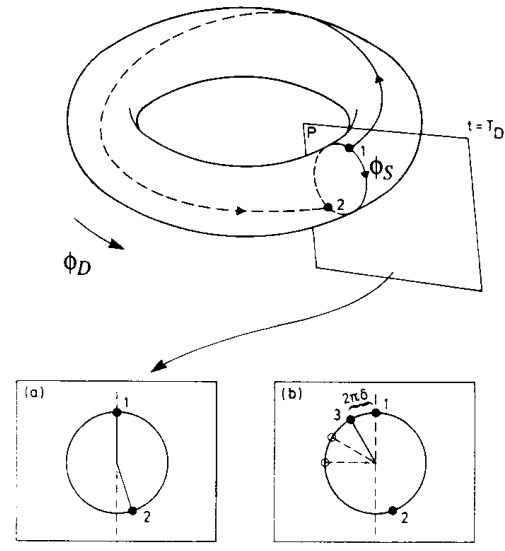

Figure 1. The dynamics in phase-space for the case $k=1$ where $\omega_{S} / \omega_{D} \approx 1 / 2 . \delta$ is the detuning between $\omega_{S}$ and $1 / 2 \omega_{D}$. The motion in a Poincaré section is also shown.

$0018-9464 / 89 / 0300-1408 \$ 01.00 \odot 1989$ IEEE 
therefore always measured with respect to the nearest resonance frequency, $\omega_{S} / \omega_{D}=1 / 2,1,3 / 2, \cdots$.

It can be shown that the asymptotic behavior around the fix points is described by the following complex equation (Ref.(9))

$$
\xi_{n+1}=\left(\lambda+(-1)^{n k} A_{1} e^{i \pi \delta}\right) \xi_{n}+(-1)^{n k} A_{0} e^{i \pi n \delta},
$$

where $\xi_{n}$ is the deviation from the fix point on the center manifold. Here $A_{0}$ and $A_{1}$ are constants which are small compared to 1, and are assumed to be proportional to the amplitude of the perturbation $s(t)$ in Eq. (1).

The solutions to this equation define the resonances in the system. There is one fix point for even $k$ and two for odd $k$.

The stability of the fix points may now be evaluated. The way to do this is to look at the asymptotic behavior around the fix point(s). The deviation from the fix point is defined as $\Delta \eta_{n}=\eta_{n}-\eta^{f i x}$. This leads to the following equation for the asymptotic behavior:

$\Delta \eta_{n+1}=\left(\lambda e^{-i \pi \delta}+(-1)^{n k} A_{1}\right) \Delta \eta_{n}$.

The two cases 1) $k$ even and 2) $k$ odd will be treated separately.

1) $k$ even:

The fix point is stable if $\left|\left(\lambda e^{-i \pi \delta}+(-1)^{n k} A_{1}\right)\right|<1$. At the verge of stability we have $-\lambda-A_{1} \cos \pi \delta \approx 1$ where $\lambda$ is the eigenvalue corresponding to the fix point $(\lambda=-1$ at the unperturbed bifurcation point). For $\lambda=-1-\mu$, where $\mu$ is a bifurcation parameter, we get the an expression for the shift of the original bifurcation point, $\Delta \mu=\mu-\mu_{0}$,

$$
\Delta \mu \propto A_{1} \cos \pi \delta
$$

$A_{1}$ is proportional to the amplitude of the periodic perturbation. The change in stability therefore depends on the sign of the amplitude and thereby on the phase of the perturbation. We see that $i t$ is not possible to make a definite prediction for the change of stability when the periodic perturbation is close in frequency to the resonance frequencies $\omega_{S} / \omega_{D}=1,2,3, \cdots$. ( $k$ even $)$.

2) $k$ odd: In this case the sign of $A_{1}$ changes for each iteration so the stability has to be investigated for every other iteration. The criterion for stability becomes $\left|\left(\lambda^{2} e^{-i 2 \pi \delta}+A^{2_{1}}\right)\right|<1$. Again, at the verge of stability: $\lambda^{2}-A^{2} \cos 2 \pi \delta \approx 1$. This leads to the following equation for the shift of the bifurcation point

$$
\Delta \mu \propto A_{1}^{2} \cos 2 \pi \delta
$$

From this we get the important result: When the periodic perturbation is close in frequency to the resonance frequencies $\omega_{S} / \omega_{D}=1 / 2,1,3 / 2, \cdots$, a periodic perturbation will always stabilize the system against a perioddoubling bifurcation, with a shift of the bifurcation point proportional to the square of the amplitude of the perturbation. Increasing the detuning $\delta$ diminishes the effect, i.e. $\Delta \mu$ decreases for increasing $\delta$.

Another important property of a period-doubling

bifurcation is its ability to amplify perturbations. Moving close to the bifurcation point, the fix points will move far away from the original unperturbed fix point which corresponds to a dynamic amplifying process or a resonance in the dynamical system: $\lambda=\left(1+A_{1}^{2}\right) \cos 2 \pi \delta$ yielding $2 \Delta \mu \approx A_{1}^{2}-2(\pi \delta)^{2}$. In general the resonance differs a little from the bifurcation (see Eqs.(7) and (8)).

We note that the bifurcation signal will be at the frequency $\omega_{D} / 2$, while the resonance will be at $\omega_{S}$. Due to the non-linearity the signal at $\omega_{D}$ (when close to $\omega_{D} / 2$ ) will couple to $\omega_{D} / 2$, in particular in the presence of noise. In the power spectrum around the period-doubled signal, the resonance will always appear at a value of $\mu$ below the bifurcation point, thereby making it impossible to observe the actual bifurcation by this method.

\section{Numerical calculations}

In order to illustrate the features of the above theory, the differential equation for a small microwave-driven Josephson tunnel junction was integrated numerically. The governing second order nonlinear differential equation is

$$
\ddot{\phi}+\alpha \dot{\phi}+\sin \phi=a_{D} \sin \omega_{D} t+a_{S} \sin \omega_{S} t+i+i_{N}(t) \text {. (9) }
$$

Here $\phi$ is the quantum-mechanical phase difference across the junction and $\alpha$ is the damping parameter $\left(\alpha=1 / \sqrt{\beta_{C}}\right.$, where $\beta_{C}$ is the McCumber parameter for the junction). $a_{D}=A_{D} / I_{C}$ and $a_{S}=A_{S} / I_{C}$ are the normalized amplitudes of the driving and resonant perturbing signals (normalized to the critical current of the junction, $\left.I_{C}\right) \cdot \omega_{D}$ and $\omega_{D}$ are the corresponding frequencies (normalized to the junction maximum plasma frequency $\omega_{p}=\left(2 e I_{C} / \hbar C\right)^{1 / 2}$ where $C$ is the junction capacitance). $i=I_{D C} / I_{C}$ is the normalized dc bias and $i_{N}=I_{N} / I_{C}$ the normalized white noise current that arises from the damping of the junction. We note that this equation also describes a driven, damped pendulum subjected to a periodic perturbating force and to a stochastic (Brownian) force term.

In order to integrate Eq.(9) we have used a fourth order Runge-Kutta method with 32 points per period of the driving signal. The motion of $\phi$ was followed over 256 periods of the drive and the first 128 periods were discarded in order to remove transients. Throughout our calculations the following fixed parameter values were used $\alpha=0.2, \omega_{D}=1.6$ and $i=0.73 . a_{D}$, and $a_{S}$ were varied. The amplitude of the driving force, $a_{D}$, was chosen as the control parameter $(\mu)$ of the system and it was chosen so the system would be near a period-doubling bifurcation.

The results of digital calculations for $\omega_{S} / \omega_{D} \approx 1 / 2$ and three different values of $\delta$ are shown in Fig. 2 (here we neglect $\left.i_{N}(t)\right)$. The shift of the bifurcation point as measured by the change in the drive amplitude, $a_{D}$, is plotted versus the perturbation amplitude, $a_{S}$. From Fig. 2 it is seen that for the calculated values the quadratic scaling law predicted by Eq.(8), $\Delta a_{D} \propto a_{S}^{2}$, holds as long as the perturbation amplitude is small. Above a certain level of perturbation which depends on the relative detuning, $\delta$, the relationship between $a_{D}$ and $a_{S}$ becomes almost linear.

Analog simulations of Eq.(9) (using a Magerlein type analog circuit) confirmed the quadratic scaling. We also used analog simulations to investigate how variations in the relative detuning tuning, $\delta$, affect the perturbation amplitude which is necessary in order to produce a constant small shift of the bifurcation. The results confirmed the prediction of Eq.(8): $a_{D} \propto \delta$ for fixed $\Delta \mu$.

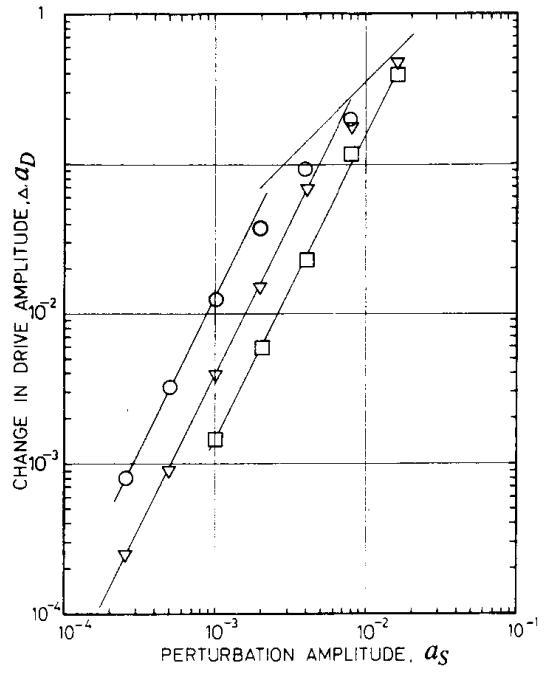

Figure 2. Results of numerical calculations for the change in the drive amplitude necessary to reach the bifurcation point, $\Delta A_{D}$, versus the perturbation amplitude for three values of the relative detuning, $2 \delta / \omega_{D}$ : circles $-1 / 256$; triangles $-1 / 128$ and squares $-1 / 64$. The fitted lines have slopes of 2 and 1 . 


\section{Experimental results on Josephson junctions}

\section{Experimental technique:}

The experiments were carried out on $\mathrm{Nb}-\mathrm{NbOx}-\mathrm{Pb}$ tunnel junctions of the overlap type. The junction areas were about $30 \times 30 \mu \mathrm{m}^{2}$ with capacitances around $90 \mathrm{pF}$, maximum plasma frequencies, $\omega_{p} / 2 \pi$, in the range 10 to $25 \mathrm{GHz}$, and Josephson penetration depth, $\lambda_{J}=50-120 \mu \mathrm{m}^{2}$ (the junction dimensions were small compared to $\lambda_{J}$ ).

In order to minimize the effect of room temperature noise and interference from external noise sources on the junction, all leads connected to the junction were carefully shielded and filtered. The ambient magnetic field was reduced to a few milligauss by two concentric shields of high permeability metal $(\mu$-metal) around the cryostat. A small magnetic field generated by a copper wire-wound solenoid could be applied in the plane of the junction in order to be able to vary $I_{C}$ and thereby $\omega_{p}$. The entire experimental set-up was placed in an rf-shielded room.

The microwave coupling to the junction was established by an inverted microstrip structure (Fig. 3). The glass substrate with the junction and its coupling circuit was mounted in a temperature-stabilized metal box with a large heat capacity which in turn was placed in a vacuum can immersed in a pressure-regulated liquid-helium bath. The temperature of the junction could be held constant to within $\pm 10 \mu K$.

The junction was driven by a monochromatic microwave signal, $A_{D} \sin \omega_{D} t$, from a synthesizer source in the frequency range $16-20 \mathrm{GHz}$ (line-width $<100 \mathrm{~Hz}$ ) coupled to the junction through a direct inverted microstrip line seen in Fig. 3. A possible period-doubled (half-harmonic) signal generated in the junction (near the plasma frequency) was coupled out through the same microstrip connection to a lownoise field-effect transistor (FET) amplifier which was followed by a digital storage spectrum analyzer (with a minimum resolution bandwidth of $100 \mathrm{~Hz}$ ). To avoid saturation of the amplifier by the pump signal, low-pass filters were inserted between the junction and the amplifier.

The perturbation signal, $A_{S} \sin \omega_{S} t$, generated in a second microwave source was coupled into the junction via an "L"-shaped microstrip antenna line (see Fig. 3). The frequency of the perturbation signal was slightly detuned (by 50 $\mathrm{kHz}$ to $100 \mathrm{MHz}$ ) from the period-doubled signal frequency at $1 / 2 \omega_{D}$. Since $1 / 2 \omega_{D} \approx 10 \mathrm{GHz}$ the relative detuning was therefore in the range $5 \times 10^{-6}$ to $5 \times 10^{-2}$. The line-width of the perturbation signal was $2 \mathrm{kHz}$.

The microwave coupling to the junction was investigated by using the junction itself as a detector. The suppression of the critical current of the junction was measured as a

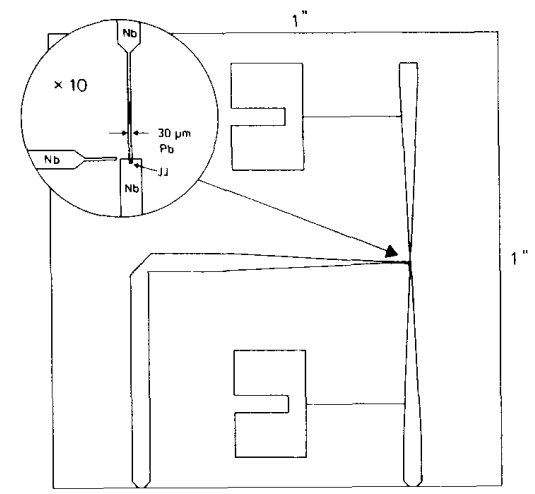

Figure 3. Sample geometry including the microwave coupling circuit and the overlap $\mathrm{Nb}-\mathrm{NbOx}-\mathrm{Pb} \quad 30 \times 30 \mu \mathrm{m}^{2}$ Josephson tunnel junction. A copper ground plane is placed app. $400 \mu \mathrm{m}$ above the glass substrate with the $\mathrm{Nb}$ thin-film microstrip circuit. The contact pads for the dc bias leads are also shown. function of the frequency and the power applied from the two sources. From such measurements we deduced that the microwave coupling both in the vicinity of the pump frequency and of its half-harmonic was strongly frequency dependent but always weak. By a comparison between the measured values of the critical current of the junction as a function of the applied microwave power, $I_{C}\left(A_{D}\right)$ and $I_{C}\left(A_{S}\right)$, and the results of numerical simulations based on the resistively-shunted junction model including noise (Eq.(9)) we were able to determine the amplitude of the microwave currents through the junction, $A_{D}$ and $A_{S}$.

In order to characterize the white noise term $\left(I_{N}(t)\right.$ in Eq.(9)), the effective noise temperature affecting the junction was measured in situ by recording the noise-perturbed distribution of the critical current for a large number of switching events, $P\left(I_{C}\right)$, in a low-noise data acquisition system (the switching was induced by sweeping the dc bias thereby tracing out the hysteretic I-V curve of the junction). Assuming a white noise spectrum such a measurement of the noise currents (primarily around the junction plasma frequency) yielded an effective noise temperature of $12 \mathrm{~K}$ at a bath temperature of $4.2 \mathrm{~K}$.

\section{Experimental results:}

The stabilizing effect of a near-resonant periodic perturbation was clearly observed. The qualitative features of such an observation are seen in Fig. 4, where amplitude-frequency recordings from the spectrum analyzer are shown as a function of dc-bias (for fixed drive amplitude) both with and without an applied perturbation signal. The main characteristics of such experimental observations are: 1) the perturbation signal suppresses the period-doubled signal (the halfharmonic amplitude); and 2) the perturbation signal is amplified as seen by the the appearance of an idler signal at the image frequency $\omega_{i}=\omega_{D}-\omega_{S}\left(=1 / 2 \omega_{D}+\delta\right)$.

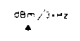

(a)
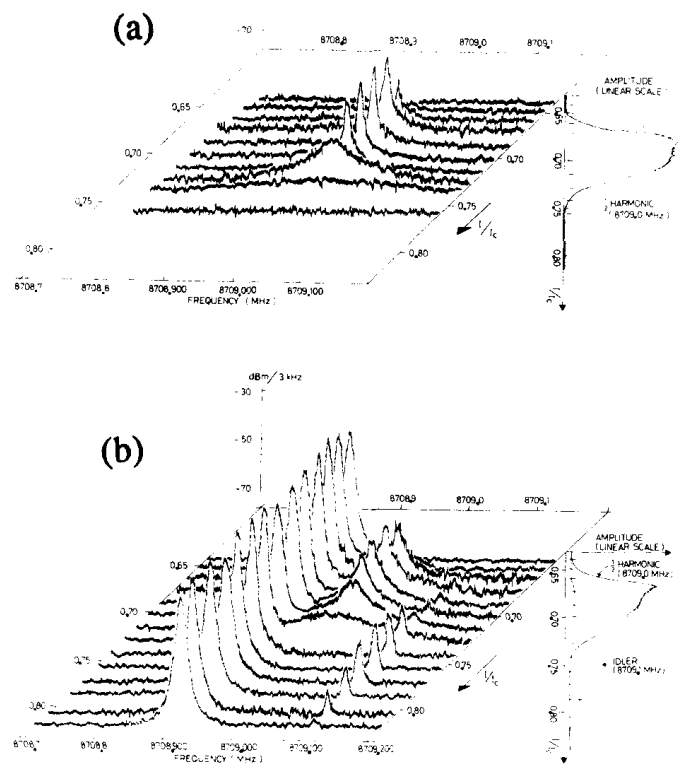

Figure 4. Experimental data for a $\mathrm{Nb}-\mathrm{NbOx}-\mathrm{Pb}$ tunnel junction showing the spectrum analyzer output vs. the dc bias both (a) without a perturbation signal applied and (b) with an applied perturbation signal detuned by $-0.1 \mathrm{MHz}$ from $1 / 2 \omega_{D}=2 \pi 8709.000 \mathrm{MHz}$. The maximum amplitudes at $1 / 2 \omega_{D}$ and at the idler (image) frequency at $8709.100 \mathrm{MHz}$ are also shown as functions of the dc bias. Both the suppression of the half-harmonic signal and the amplification are seen in this figure. 
We note that the microwave set-up used in this work did not provide isolation between the input and the output of the junction and we have therefore not been able to measure the amplification in the system. The appearance of the idler signal is, however, indicating that the junction is working as an amplifier.

Figure 5 shows how the onset of the period-doubled signal is shifted towards higher values of the drive amplitude when the amplitude of the perturbation signal is increased, i.e. the bifurcation point is moved towards larger $A_{D}$ values.

To find a possible characteristic scaling behavior of the shift of the bifurcation point we again use the amplitude of the drive signal as the non-linearity parameter, $\mu$. From the experimental curves like the set reproduced in Fig. 5 we calculate the shift of the bifurcation point, $\Delta \mu=\Delta A_{D}$ as a function of the perturbation amplitude, $A_{S}$.

In Fig. 6 the bifurcation point shift found in this way is

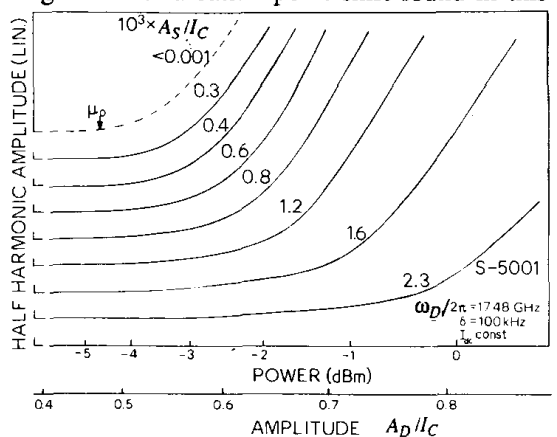

Figure 5. Smoothed experimental recordings of the half-harmonic amplitude (lin. scale) versus the normalized drive amplitude, $A_{D} / I_{C}$, for a number of different values of the normalized perturbation signal amplitude, $A_{S} / I_{C}$. The dc bias is constant. $\mu_{0}$ is the unperturbed bifurcation point, i.e. the threshold for the onset of the period-doubled signal (the half-harmonic) without perturbation.

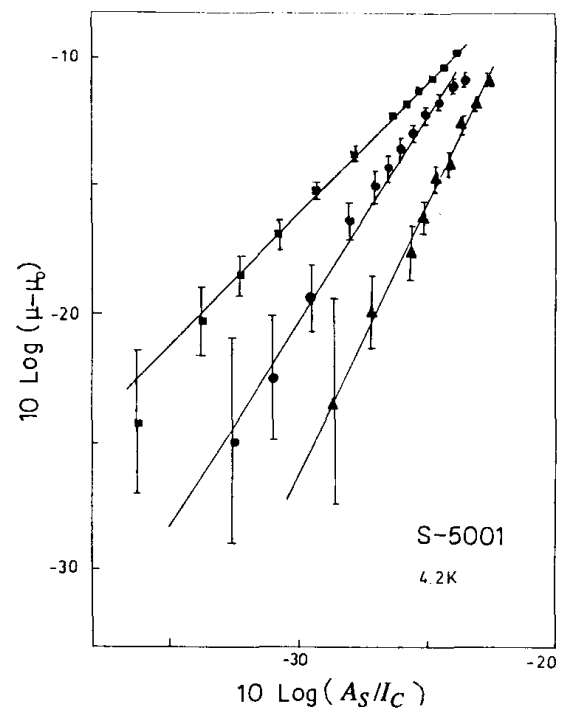

Figure 6. Experimental data for a $\mathrm{Nb}-\mathrm{NbOx}-\mathrm{Pb}$ tunnel junction at $4.2 \mathrm{~K}$. The $\log$-log plot shows the measured scaling behavior of the shift of the bifurcation point (as defined by the minimum drive amplitude for which the period-doubled signal is observed, see Fig. $5)$ as a function of the normalized perturbation amplitude, $A_{S} / I_{C}$. for three different values of the detuning, $\delta$ : squares $0.1 \mathrm{MHz}$; cir. cles $10 \mathrm{MHz}$ and triangles $40 \mathrm{MHz}$. The value of $1 / 2 \omega_{D} / 2$ is $2 \pi \cdot 8709.000 \mathrm{MHz}$. The three straight lines are leastsquares fits to the data points. The slopes give the following scaling exponent: squares 1.0 ; circles 1.6 and triangles 2.1 . plotted versus the perturbation amplitude for three different values of the frequency detuning. From such doublelogarithmic plots we have found a linear $\Delta \mu$ vs. $A_{S}$ dependence for small detuning and a quadratic scaling behavior for large detuning: $\Delta \mu \propto A_{S}^{2}$.

Finally, for fixed $\delta$ we have investigated how the necessary perturbation amplitude depends on the detuning. It turns out that such measurements are very difficult to perform on a Josephson junction due to the strong frequency dependence of the microwave coupling to the junction. Technically it is not easy to vary the perturbation frequency over a wide band, say from $0.1 \mathrm{MHz}$ to $100 \mathrm{MHz}$ (corresponding to a relative detuning from $10^{-5}$ to $10^{-3}$ ) without strongly varying the perturbation amplitude in the junction. We have therefore not at present been able to test the theoretical prediction for the scaling law for $A_{S}(\delta)$ for fixed $\Delta A_{D}$

\section{Discussion and conclusion}

The stabilization of a near-resonant perturbation signal against the onset of a period-doubling bifurcation which is predicted by the theory and confirmed through numerical calculations and analog simulations has been observed in small Josephson tunnel junctions biased on the supercurrent.

Theoretically, the strength of this stabilization which may be characterized by the shift of the bifurcation point has been shown to depend on the perturbation amplitude according to a characteristic quadratic scaling law, i.e. $\Delta \mu \propto A_{S}^{2}$, where we have used the change in the drive amplitude, $\Delta A_{D}$, as a measure of the shift of the bifurcation point (i.e. we use $A_{D}$ as the non-linearity parameter of the system).

In the experiments the shift in the bifurcation point was measured by the change of the minimum value of the drive amplitude for which a period-doubled signal could be observed above the background noise level in the system (Fig. 5 ). This shift was found to scale linearly with the perturbation amplitude for small frequency detuning and quadratically for large detuning of the perturbation signal (Fig. 6). The same behavior was found in the numerical calculations (Fig. 2). For small values of the detuning, the relative perturbation becomes large, and non-linear effect will dominate and the theory breaks down. For small values of the relative perturbation amplitude the theory can be applied, and good agreement between the theoretical prediction and experimental results is found: $\Delta \mu \propto A_{S}^{2}$.

\section{Acknowledgment}

We thank I. Rasmussen, S. Hjorth and J. L. Skov for the sample fabrication and T. Holst for the analysis of the experimental noise data. This work was partly supported by grants from the Danish Natural Science Research Council.

\section{References}

(1) Kurt Wiesenfeld and Bruce McNamara, Phys. Rev. Lett., Vol. 55 . p. 10,1985 .

(2) Kurt Wiesenfeld and Bruce McNamara, Phys. Rev., Vol. A33 , p. $629,1986$.

(3) Paul Bryant and Kurt Wiesenfeld, Phys. Rev., Vol. A33 , p. 2525 , 1986.

(4) Kurt Wiesenfeld, J. Stat. Phys., Vol. 28 , p.1071, 1985.

(5) H. Svensmark, J. Bindslev Hansen, and and N. F. Pedersen, Phys. Rev., Vol. A35 , p.1457, 1987.

(6) A.D. Smith, R.D. Sandell, J.F. Burch and A.H. Silver, IEEE Trans. Magn., Vol. MAG-21 , p. 1022, 1987.

(7) H.K. Olsson and T. Claeson, Jpn. J.Appl. Phys., Vol. 26 , p. 1547 , 1987.

(8) J. Guckenheimer and P. Holmes, Nonlinear Oscillations, Dynamical Systems and Bifurcations of Vector Fields. New York, Springer, 1984.

(9) H. Svensmark and M.R. Samuelsen, Phys. Rev., Vol. A36 , p. 2413, 1987.

(10) G. Friis Eriksen, J. Bindslev Hansen, M. R. Samuelsen and H. Svensmark, to be published. 